\title{
Concerted Dihedral Rotations Give Rise to Internal Friction in Unfolded Proteins
}

\author{
Ignacia Echeverria, ${ }^{\dagger}$ Dmitrii E. Makarov, ${ }^{\ddagger}$ and Garegin A. Papoian* ${ }^{\dagger}$ \\ ${ }^{\dagger}$ Department of Chemistry and Biochemistry and Institute for Physical Science and Technology, University of Maryland, College \\ Park, Maryland 20742, United States \\ ${ }^{\ddagger}$ Department of Chemistry and Institute for Computational Engineering and Sciences, University of Texas at Austin, Austin, Texas \\ 78712, United States
}

Supporting Information

ABSTRACT: Protein chains undergo conformational diffusion during folding and dynamics, experiencing both thermal kicks and viscous drag. Recent experiments have shown that the corresponding friction can be separated into wet friction, which is determined by the solvent viscosity, and dry friction, where frictional effects arise due to the interactions within the protein chain. Despite important advances, the molecular origins underlying dry friction in proteins have remained unclear. To address this problem, we studied the dynamics of the unfolded cold-shock protein at different solvent viscosities and denaturant concentrations. Using extensive all-atom molecular dynamics simulations we estimated the internal friction time scales and found them to agree well with the corresponding experimental measurements (Soranno et al. Proc.

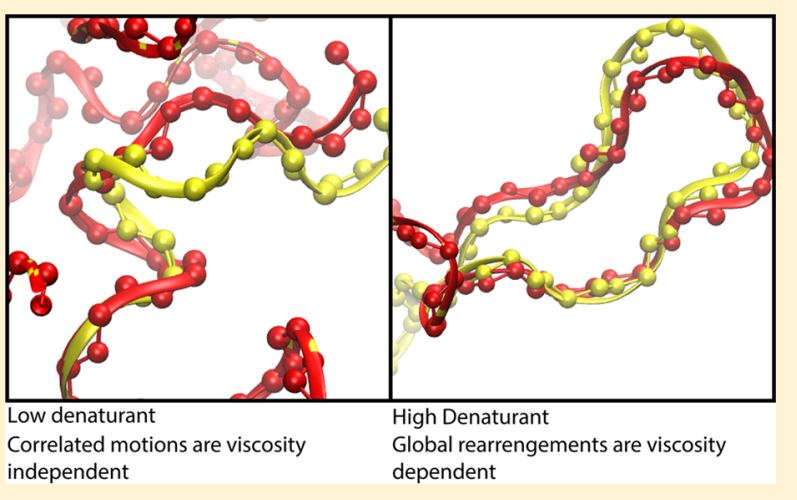
Natl. Acad. Sci. U.S.A. 2012, 109, 17800-17806). Analysis of the reconfiguration dynamics of the unfolded chain further revealed that hops in the dihedral space provide the dominant mechanism of internal friction. Furthermore, the increased number of concerted dihedral moves at physiological conditions suggest that, in such conditions, the concerted motions result in higher frictional forces. These findings have important implications for understanding the folding kinetics of proteins as well as the dynamics of intrinsically disordered proteins.

\section{INTRODUCTION}

To understand how proteins fold, it is essential to know the structural and dynamical properties of their unfolded state. For example, the time scales over which unfolded proteins undergo substantial conformational reconfiguration ultimately determine the "speed limit" or the maximum rate at which they can fold. ${ }^{1,2}$ Likewise, understanding the properties and function of the proteins that are known to be disordered in their biologically active form requires insight into the unfolded state. Despite its importance, however, atomically detailed understanding of unfolded proteins remains limited because of the inherent conformational and dynamical heterogeneity of the unfolded ensemble.

In an aqueous environment, slow, large-scale motions of an unfolded protein are necessarily diffusive. It is, however, unclear how much these motions are controlled by viscous friction exerted by the solvent, as compared to the effect of the inherent intramolecular energy landscape. Experimentally, this question has been addressed by measuring the viscosity dependence of various time scales of protein dynamics (e.g., the folding time or reconfiguration time in the unfolded state). When the relevant time $\tau$ is found to depend linearly on the solvent viscosity $\eta$, i.e.,

$$
\tau=a \eta+\tau_{\mathrm{i}}
$$

then the zero-viscosity intercept $\tau_{\mathrm{i}}$ is usually attributed to internal friction. However, the molecular mechanisms giving rise to the time scale $\tau_{\mathrm{i}}$ are not understood. Moreover, eq 1 is entirely phenomenological, as experiments, ${ }^{3-7}$ simulations, $^{8}$ and theoretical studies ${ }^{9-12}$ suggest that nonlinear viscosity dependencies are possible (depending on the nature of the time scale $\tau$ in question).

A recent study of the reconfiguration dynamics of the unfolded cold shock protein (CSP) ${ }^{6}$ offers a step toward the microscopic view of internal friction. In addition to observing that the characteristic reconfiguration time is well described by eq 1 , the authors ${ }^{6}$ probed the relaxation spectrum of the unfolded protein by measuring relaxation times within pairs of different residues. Their data supported a well established coarse-grained model of polymer dynamics, known as the Rouse model with internal friction (RIF), ${ }^{13-16}$ as a quantitative description of the dynamics in the unfolded state. Similarly to eq 1, RIF accounts for internal friction effects by introducing a single time scale $\tau_{\mathrm{i}}$. RIF, however, makes a more detailed prediction regarding the entire relaxation spectrum of the chain.

Received: $\quad$ March 26, 2014 
Specifically, the relaxation time of mode $n\left(\tau^{(n)}\right)$ is incremented by $\tau_{\mathrm{i}}$

$$
\tau^{(n)} \rightarrow \tau^{(n)}+\tau_{\mathrm{i}}
$$

and thus internal friction has a more significant effect on faster relaxation modes. Internal friction effects were found to be significant at low denaturant concentrations, where the unfolded chain is more compact, while negligible at highly denaturing conditions.

The original justification of RIF goes back to Kuhn's view of hopping over barriers for hindered rotations, ${ }^{13,17-19}$ where the internal friction time $\tau_{\mathrm{i}}$ can be related to the hopping rates ${ }^{13}$ and it is independent of the chain length. While possibly adequate for simple homopolymers, such a view might be a gross oversimplification for proteins, where, e.g., hydrogen bonding, hydrophobic effect, and sequence-specific interactions likely contribute to the overall conformational statistics and dynamics. Moreover, this view does not explain why and how internal friction depends on the denaturant concentration.

Here, we report on atomistic simulations of the unfolded CSP performed at different solvent viscosities and denaturant concentrations. Our simulations are in agreement with the coarse-grained RIF picture, thus allowing us to obtain a first principle estimates of $\tau_{\mathrm{i}}$, which, consistent with experimental observations, are found to increase as the denaturant concentration is decreased. To elucidate the molecular origins of the internal friction time scale $\tau_{\mathrm{i}}$, we carried out a detailed analysis of dihedral dynamics. This analysis supports the view that internal friction predominantly arises from hops in dihedral space. Although this process is reminiscent of the Kuhn mechanism, it is nevertheless distinct because it crucially depends on the chain compactness and involves multiresidue, correlated dihedral angle hopping dynamics.

\section{SIMULATION DETAILS}

Molecular Dynamics (MD) Simulations. All of our simulations were carried out using the GROMACS $4.5^{20} \mathrm{MD}$ software, the amber03 ${ }^{21}$ force field, and the SPC/E water model. Starting from the NMR structure of the Thermotoga maritima CSP (pdb access code 1G6P), ${ }^{22}$ the initial model was built by solvating the protein in a box of water molecules of dimensions $7 \times 7 \times 7 \mathrm{~nm}^{3}$. Counterions were added using the genion module of Gromacs, which randomly replaces water molecules with counterions in favorable locations determined by computing the electrostatic potential at the insertion site. ${ }^{20}$ The system was minimized using, first, the steepest-descent and then the conjugate gradient method, for 4000 steps in each case. The protein was then unfolded by gradually heating the system from 100 to $800 \mathrm{~K}$ and back to $300 \mathrm{~K}$ over a 1 ns simulation. The system was then equilibrated at $300 \mathrm{~K}$ (for 500 ps) in the NPT ensemble. Periodic boundary conditions were used in all our calculations, and long-range electrostatics were treated with the particle mesh Ewald method. ${ }^{23}$ The cutoff distance for nonbonded Coulomb and Lennard-Jones interactions was set to $0.9 \mathrm{~nm}$. All bonds were constrained using the LINCS algorithm. ${ }^{24}$ Production simulations were performed at a constant temperature (of $300 \mathrm{~K}$ ) and pressure by using the velocity rescaling thermostat the and Parrinello-Rahman barostat. The pressure was set to $0.138 \mathrm{~atm}$, in order to mimic the experimental conditions. ${ }^{6}$

Modified Solvent Viscosity. One of the most common methods of quantifying internal friction is to vary the solvent viscosity $(\eta) .^{6-8}$ In the current work, we used the method of rescaling the solvent molecules mass, which provides a simple and effective way to modify the solvent viscosity, ${ }^{8}$ accelerating all the transport properties (e.g., the folding times), without altering the equilibrium properties of the protein ${ }^{8,25}$ (see Supporting Information, Figure S8). Simulations were performed at three different denaturant (guanidinium chloride, $\mathrm{GdmCl})$ concentrations $(0,2$, and $4 \mathrm{M})$ and at three different solvent viscosities $\left(\eta=0.5,1\right.$, and $\left.1.4 \eta_{0}\right)$, where $\eta_{0}$ is the viscosity of water $(1 \mathrm{mPa} \cdot \mathrm{s})$. The integration time step for the simulations was selected according to the solvent viscosity and was equal to $0.25 \mathrm{fs}$ at $0.5 \eta_{0}$ and 2 fs at $1.0 \eta_{0}$ and $1.4 \eta_{0}$. Production runs for each combination of solvent viscosity and denaturant concentration involved trajectories spanning 800$1200 \mathrm{~ns}$, with total simulation time of $9 \mu \mathrm{s}$.

Modified Dihedral Potentials. The potential energy function describing the backbone dihedral angles has the form:

$$
V_{\text {dihe }}(\theta)=\frac{V_{n}}{2}[1+\cos (n \theta-\gamma)]
$$

where $\theta$ is the dihedral angle (either $\phi$ or $\psi$ ) and $V_{n}$ is the corresponding force constant. The phase angle $\gamma$ takes values of either $0^{\circ}$ or $180^{\circ}$, and $n$ is an integer that determines the periodicity of the potential. ${ }^{21}$ To explore the connection between internal friction and the dihedral energy landscape, we repeated our simulations, in the zero-denaturant case, using a softer dihedral potential, with all dihedral barriers reduced by a factor of 2 , i.e., $V_{n} \rightarrow V_{n} / 2$.

\section{RESULTS AND DISCUSSION}

Lowering the Denaturant Concentration Leads to More Compact States. Simulations of the unfolded CSP in explicit solvent at different concentrations of $\mathrm{GdmCl}$ show that the conformational ensemble sampled by the unfolded protein depends on the denaturant concentration, exhibiting more compact configurations at lower denaturant concentrations. Specifically, the average radius of gyration $\left(R_{\mathrm{g}}\right)$ decreases as the $\mathrm{GdmCl}$ concentration is decreased (Figure 1 ), an observation

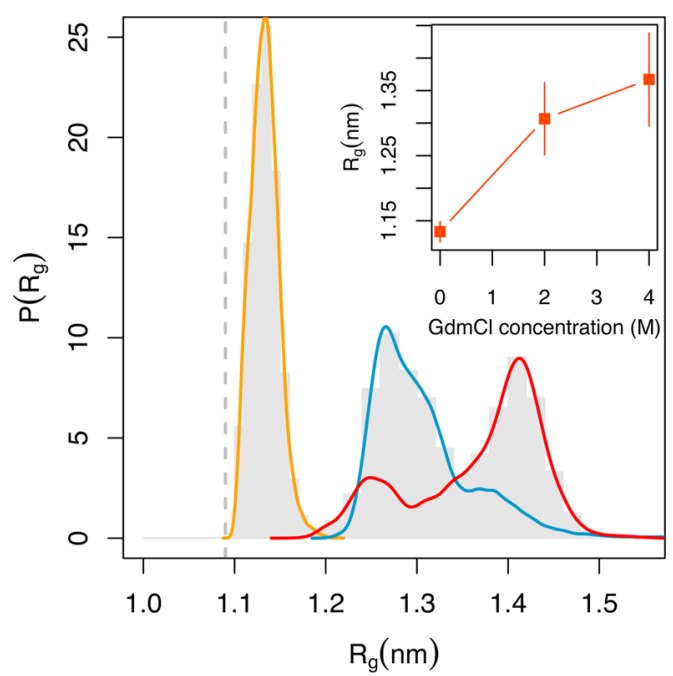

Figure 1. Probability distribution of the radii of gyration $\left(R_{\mathrm{g}}\right)$ at different denaturant concentrations: $0 \mathrm{M}$ (yellow), $2 \mathrm{M}$ (blue), $4 \mathrm{M}$ (red) $\mathrm{GdmCl}$. The vertical gray line corresponds to the $R_{\mathrm{g}}$ of the folded CSP $\left(R_{\mathrm{g}}=1.09 \mathrm{~nm}\right)$. Inset: Average $R_{\mathrm{g}}$ as a function of the denaturant concentration. Error bars represent one standard deviation. 
consistent with the experimental data of refs 6 and 7. However, the simulated $R_{\mathrm{g}}$ values are lower than the experimental ones, possibly because the force field we use is too "hydrophobic", a common problem with the majority of current force fields. ${ }^{26}$ The effects of the dyes used to probe intramonomer distances experimentally may further contribute to this discrepancy ${ }^{27}$ (see Supporting Information for further details).

Characteristic Times of Dihedral Dynamics Are Comparable to Those of Internal Friction. In the context of protein dynamics, a friction mechanism analogous to Kuhn friction can be described as follows: The geometry of the protein backbone is largely specified by just two degrees of freedom per residue: the dihedral angles $\phi$ and $\psi$. Because of steric constraints these torsional degrees of freedom can access only certain values, thus creating an effectively discrete backbone conformational space. The dihedral angles change via hops among distinct isomeric states, separated by energy barriers, thereby contributing to the overall ruggedness of the free energy landscape on a local scale.

To examine the role of this mechanism in the dynamics of the unfolded state we computed the autocorrelation function of the dihedral angles $\left\langle\cos \left[\theta_{n}(t)-\theta_{n}(t+\tau)\right]\right\rangle$, as a function of the lag time $\tau$ (see Supporting Information for details). For notational simplicity, we have relabeled the dihedral sequence $\left(\phi_{1}, \psi_{1}, \phi_{2}, \psi_{2}, \ldots\right)$ as $\left(\theta_{1}, \theta_{2}, \theta_{3}, \theta_{4}, \ldots\right)$. When averaged over all dihedrals, this autocorrelation function decays on a time scale of tens of nanoseconds (Figure 2A), which is slower than the dihedral relaxation times observed for folded proteins and short peptides. $^{28,29}$ Remarkably, the dihedral relaxation time $\left(\tau_{\mathrm{R}}^{\mathrm{d}}\right)$ is viscosity independent at 0 and $2 \mathrm{M} \mathrm{GdmCl}$ concentration (Figure 2B), suggesting that at no and low denaturant
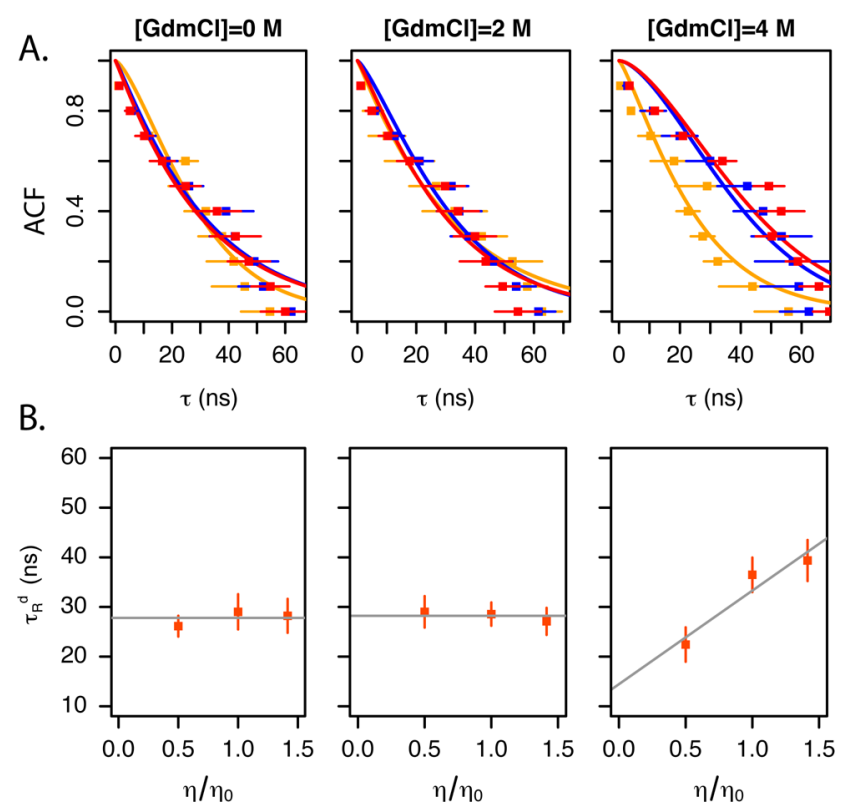

Figure 2. Dihedral angle relaxation times. (A) Dihedral angle autocorrelation function (averaged over all protein dihedrals, filled squares) at different denaturant concentrations and solvent viscosities: $\eta / \eta_{0}=0.5$ (yellow), $\eta / \eta_{0}=1$ (blue), $\eta / \eta_{0}=1.41$ (red). Error bars correspond to one standard deviation. Lines represent stretched exponential fits as described in the Supporting Information. Fits are included only for visualization purposes. (B) Average dihedral relaxation times $\left(\tau_{\mathrm{R}}^{\mathrm{d}}\right)$. The intercepts at $\eta / \eta_{0}=0$ are $27.8 \pm 3.0$, $28.2 \pm 2.7$, and $18.8 \pm 6.0 \mathrm{~ns}$ at 0,2 , and $4 \mathrm{M} \mathrm{GdmCl}$, respectively. concentration the local reorganization of the backbone is not significantly coupled to the solvent dynamics. ${ }^{30}$ Furthermore, typical dihedral relaxation time scales are comparable with those of internal friction observed in, ${ }^{6}$ suggesting that, indeed, the friction mechanism determined by dihedral barrier crossing events may, at least in part, account for the experimentally observed internal friction effects.

Increased Number of Concerted Dihedral Hops Is Coincidental with the Onset of Internal Friction. Among different types dihedral transitions, correlated motions are particularly important in protein dynamics. ${ }^{28,31,32}$ Indeed, in contrast to single dihedral changes, which may involve the pivoting of two large polypeptide segments around a single rotation axis and result in large viscous drag and/or steric clashes, crankshaft-like transitions involve a correlated change in two or more dihedral angles, resulting in relatively localized chain rearrangements. ${ }^{28,31,32}$

In order to explore the dynamical correlations among the dihedrals, we further identified correlated rotations as the events where, say, the dihedrals $n$ and $m$ undergo transitions separated by less than some specified (and short) time interval $\delta t$ (see Supporting Information). Here, the chosen $\delta t$ is much shorter than the average lag time between consecutive jumps of individual dihedral angles (see Supporting Information, Figure S5). Each dihedral rotation is, therefore, viewed as part of a correlated move or not. The fraction of dihedral rotations belonging to a correlated move increases when the denaturant concentration is decreased, with more than $50 \%$ of the isomeric transitions occurring as part of correlated motions at $0 \mathrm{M}$ $\mathrm{GdmCl}$ (Figure 3). Moreover, while at a high denaturant

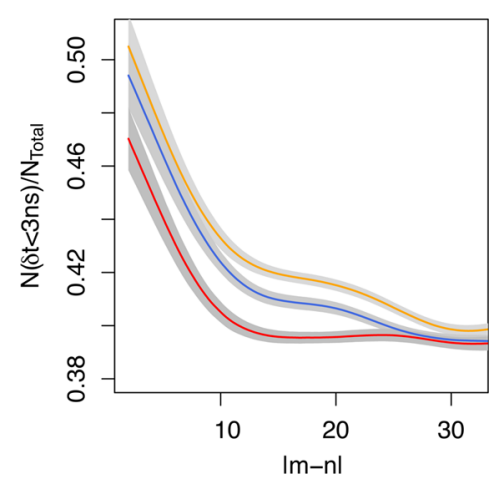

Figure 3. Correlation length for dihedral rotations. Fraction of dihedral hops of a torsion angle $m$ that occur within $3 \mathrm{~ns}$ of an isomeric transition of an angle $n(N(\delta t<3 \mathrm{~ns}))$, normalized by the total number of transitions $N_{\text {Total, }}$ as a function of $|m-n|$. Same color scheme as above. Gray shaded areas correspond to one standard deviation. Note that $n$ and $m$ enumerate dihedrals rather than residues so $|n-m|$ should be divided by 2 in order to obtain the sequence separation.

concentration $(4 \mathrm{M})$ the fraction of correlated dihedral hops plateaus as $|n-m|$ exceeds 5 residues, at zero denaturant correlated hops are observed even when the dihedrals that are 15 residues apart.

These observations support the idea that local, correlated rearrangements provide a key mechanism through which the protein samples its conformational space, especially in a denaturant free environment. Moreover, in the compact molten-globule state the high number of monomers engaged in the concerted barrier-crossing transitions will result in higher effective frictional forces. ${ }^{33}$ Consequently, the contribution of 
correlated dihedral rotations to internal friction is only sizable in the collapsed state. This conclusion is consistent with the prior observations that internal friction increases upon the chain collapse, in agreement with. ${ }^{6,7,34}$ In contrast, given that single-dihedral hops are also observed at high denaturant concentrations, where internal friction is negligible, it appears plausible that their contribution to $\tau_{\mathrm{i}}$ is not significant. Nevertheless, such a contribution is hard to entirely rule out.

Intramonomer Dynamics Yields First-Principle Estimates of Internal Friction. Fluorescence resonance energy transfer (FRET)-derived protein reconfiguration times are related to the fluctuations in the distance between dye molecules attached at a specific positions along the chain, with end-labeled chains being most commonly studied..$^{5-7,35,36}$ To compare with the FRET data, we thus computed the characteristic end-to-end reconfiguration time $\tau_{\mathrm{R}}$ from the autocorrelation function of the end-to-end distance (see Supporting Information for details). As shown in Figure 4,
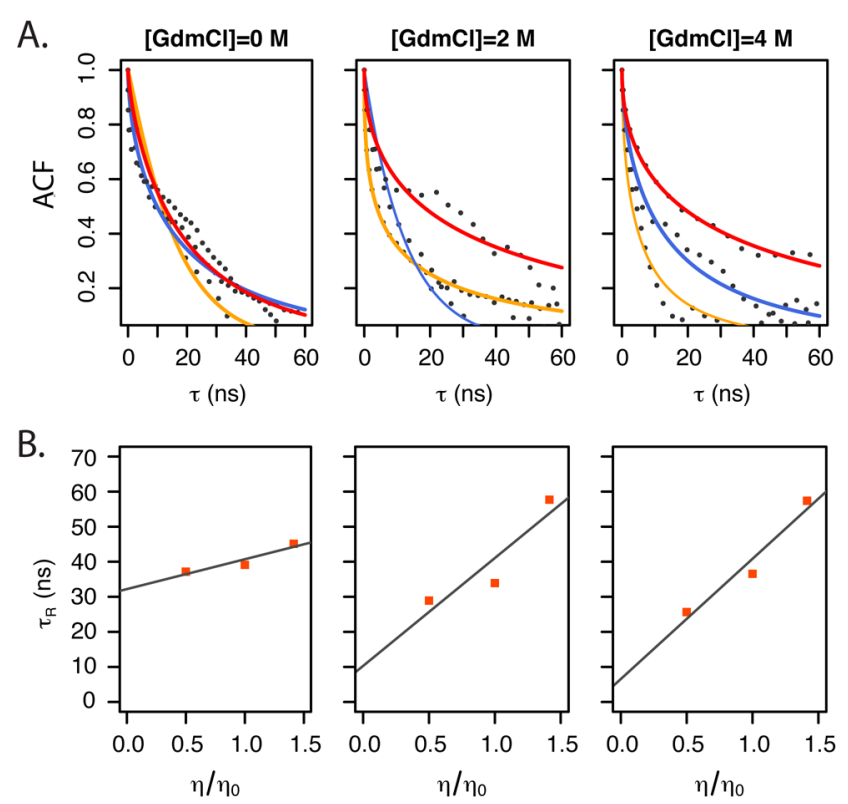

Figure 4. End-to-end distance reconfiguration times. (A) End-to-end distance autocorrelation function at different denaturant concentrations and solvent viscosities: $\eta / \eta_{0}=0.5$ (yellow), $\eta / \eta_{0}=1$ (blue), and $\eta / \eta_{0}=1.41$ (red). Gray dots are raw simulation data, while colored lines are stretched exponential fits, as described in the Supporting Information. Fits are included only for visualization purposes. (B) Average end-to-end distance reconfiguration times $\left(\tau_{\mathrm{R}}\right)$ plotted as a function of solvent viscosity at different denaturant concentrations. The intercepts at $\eta / \eta_{0}=0$ are $32.2 \pm 1.8,10.3 \pm 4.8$, and $6.6 \pm 4.6 \mathrm{~ns}$ at 0,2 , and $4 \mathrm{M} \mathrm{GdmCl}$, respectively.

this autocorrelation function and, consequently, the reconfiguration time, is viscosity dependent at all denaturant concentrations. This dependence becomes more pronounced as the denaturant concentration is increased, as seen from the steeper slope at $4 \mathrm{M} \mathrm{GdmCl}$ (Figure 4B). These findings are consistent with the RIF model, which predicts that internal friction is an additive contribution to the overall end-to-end distance reconfiguration time $\left(\tau_{\mathrm{R}}\right)$ :

$$
\tau_{\mathrm{R}}=\frac{\eta}{\eta_{0}} \tau_{\mathrm{s}}\left(\eta_{0}\right)+\tau_{\mathrm{i}}
$$

where $\tau_{\mathrm{s}}$ and $\tau_{\mathrm{i}}$ are, respectively, the reconfiguration times associated with the dynamics coupled to the solvent viscosity and that independent of the solvent. The internal friction time $\tau_{\mathrm{i}}$ can thus be estimated by extrapolating $\tau_{\mathrm{R}}$ to $\eta=0$ : the resulting estimates are given in Table $1\left(\tau_{\mathrm{i}}^{\mathrm{V}}\right)$. Note that the

Table 1. Internal Friction Time $\tau_{\mathrm{i}}$ Obtained by Extrapolating the End-to-End Reconfiguration Times $\left(\tau_{i}^{\mathrm{V}}\right)$ and From CRIF Fits of Intermonomer Reconfiguration Times $\left(\tau_{\mathbf{i}}^{S}\right)$

\begin{tabular}{ccc}
$\mathrm{GdmCl}(\mathrm{M})$ & $\tau_{\mathrm{i}}^{\mathrm{V}}(\mathrm{ns})$ & $\tau_{\mathrm{i}}^{\mathrm{s}}(\mathrm{ns})$ \\
0 & $32.2 \pm 1.8$ & $20.5 \pm 8.0$ \\
2 & $10.2 \pm 4.6$ & $13.4 \pm 3.8$ \\
4 & $6.6 \pm 4.8$ & $8.1 \pm 2.1$ \\
\hline
\end{tabular}

observed values of $\tau_{\mathrm{i}}^{\mathrm{V}}$ are comparable with the dihedral angle relaxation times $\tau_{\mathrm{R}}^{\mathrm{d}}(5-35 \mathrm{~ns})$. Moreover, our estimates of $\tau_{\mathrm{i}}^{\mathrm{V}}$ are also comparable to their experimental counterparts $(20-40$ ns) measured for the same protein. ${ }^{6}$

A complementary way to estimate the internal friction from FRET experiments is to study the dependence of intramonomer reconfiguration time on their sequence location and, particularly, on the length of the chain segment flanked by the monomers. ${ }^{6,12}$ To mimic such measurements, we have computed such residue-dependent reconfiguration times $\hat{\tau}_{|j-i|}$ from the autocorrelation functions of the distances between $\mathrm{C} \alpha$ pairs $i$ and $j$, where $10<|i-j| \leq 65$ (Figure 5). Here, indices $i$ and $j$ refer to residue number.

We observe that decreasing the denaturant concentration weakens the dependence of $\hat{\tau}_{|j-i|}$ on the segment length $|i-j|$. This dependence can be fitted using a "compacted RIF" or CRIF, which is version of RIF that accounts for chain compaction upon the lowering of the denaturant concentration (see Supporting Information for further details). CRIF fits

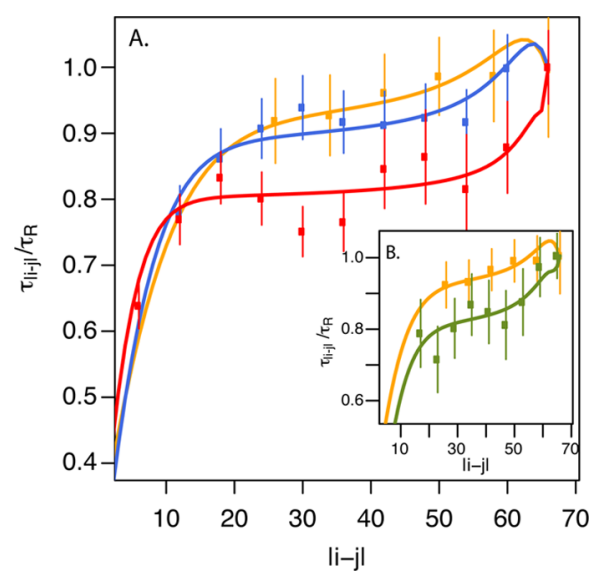

Figure 5. Reconfiguration times for polypeptide segments of different lengths. (A) Points correspond to the average reconfiguration time $\hat{\tau}_{|j-i|}$ for pairs of $\mathrm{C} \alpha$ 's that are $|i-j|$ residues apart at different denaturant concentrations: $0 \mathrm{M}$ (yellow), $2 \mathrm{M}$ (blue), and $4 \mathrm{M}$ (red) GdmCl. The time $\hat{\tau}_{|i-j|}$ was normalized by the end-to-end reconfiguration time $\tau_{\mathrm{R}}$. Lines were fitted using the CRIF model as described in the Supporting Information. (B) Yellow: same as above. Green: Same calculation as above was performed at $0 \mathrm{M} \mathrm{GdmCl}$ using a softer dihedral potential, with each rotational barrier rescaled by a factor of 0.5. The CRIF fit in the case of the soft potential yields a much weaker internal friction, $\tau_{\mathrm{i}} \sim 0.45 \tau_{\mathrm{R}}$ (where $\tau_{\mathrm{R}}$ is the end-to-end reconfiguration time), in contrast to $\tau_{\mathrm{i}} \sim 0.72 \tau_{\mathrm{R}}$ estimated for the original potential. 
provide an independent estimate of the internal friction time $\tau_{\mathrm{i}}^{S}$ as a function of denaturant (Table 1). These estimates are in reasonable agreement with the values $\tau_{\mathrm{i}}^{\mathrm{V}}$ obtained from the viscosity-dependent linear interpolation (also shown in Table 1) thus suggesting that RIF provides an internally consistent framework to account for the observed dynamics.

Energy Landscape for Dihedral Rotations Controls the Magnitude of Internal Friction. Although the similarity between $\tau_{\mathrm{i}}$ and the dihedral relaxation time is suggestive, it does not prove that internal friction originates from dihedral dynamics. To further explore the latter as the potential internal friction mechanism we repeated our simulations in the zerodenaturant case using a softer dihedral potential, with all dihedral barriers reduced by a factor of 2 (see the Simulation Details section for details). The resulting residue-dependent reconfiguration times $\hat{\tau}_{|j-i|}$ and their CRIF fit are shown in Figure 5.B. The weakening of the dihedral rotation barriers is found to lead to a marked decrease in the internal friction contribution, from $\tau_{\mathrm{i}} \sim 0.72 \tau_{\mathrm{R}}$ estimated for the original potential to $\tau_{\mathrm{i}} \sim 0.45 \tau_{\mathrm{R}}$ with the soft dihedral potential, thus providing further support to the hypothesis that a Kuhn-like dihedral hopping mechanism is responsible for internal friction.

Long-Lived Cohesive Intermolecular Interactions May Contribute to Internal Friction. An alternative explanation of internal friction is that it is caused by cohesive interactions between amino acids (such as hydrogen bonds, hydrophobic interactions, or salt-bridges). To explore this possibility we computed the distributions of times in which hydrophobic contacts and hydrogen bonds are formed and broken. These distributions are shown in the Supporting Information, indicating that, even at physiological conditions, the majority of hydrogen bonds and hydrophobic contacts are short-lived, exhibiting characteristic times shorter than $\tau_{\mathrm{i}}$ (see Supporting Information, Table 1 and Figures S9 and S10). It is, therefore, unlikely that such hydrogen bonds or hydrophobic contacts could account for the observed internal friction time scale. However, we found a number of long-lived, non-native, hydrogen bonds and hydrophobic contacts that acted as kinetic traps. In addition, since salt bridges may also slow down reconfiguration dynamics ${ }^{37}$, thereby, potentially contributing to internal friction, we also monitored their formation and breaking. However, only a small number of salt bridges were observed (see Supporting Information, Figure S13), which precludes reaching statistically significant conclusions regarding their dynamic effect. In silico mutation studies, analogous to the prior in vitro work, ${ }^{37}$ may help elucidate this issue in the future.

Finally, we explored the possibility that long-range contacts could contribute to internal friction by gating the dihedral dynamics. That is, for a dihedral rotation to occur, a contact must break in the vicinity of the atoms involved in the backbone rearrangement, releasing a segment of the chain. No significant gating by either hydrogen bonds or hydrophobic contacts was, however, found (see Supporting Information, Figure S11). We thus conclude that long-range contacts, even though they can act as kinetic traps, are not likely to provide a dominant contribution to internal friction, especially at physiological conditions.

It should be noted that intramolecular contacts, as well as other global structural features, were found to be not fully equilibrated and to lack sampling convergence. This might be an issue if equilibrium thermodynamic properties of the unfolded ensemble were desired. Nevertheless, the relaxation time scales of different local structural features, which are the focus of the present study, are unlikely to be significantly affected by this problem. Furthermore, previous MD studies of unfolded proteins have shown that, despite lack of complete sampling, relevant local and global structural properties can be obtained. $^{26}$ The accuracy of our results is also limited by the accuracy of the force field used. However, the good qualitative and quantitative agreement between our results and the experimental studies of the same protein ${ }^{6}$ suggests that the force fields used gives a reasonable description of the dynamics in the unfolded state.

\section{CONCLUSION}

The all-atom MD simulations presented here highlighted the effect of internal friction in the dynamics of unfolded proteins and demonstrated that the global dynamics of the unfolded chain, and hence the early events in the folding kinetics of proteins are determined microscopically by local motions that relax rapidly when compared to the overall folding reaction. The physical mechanism behind internal friction involves thermally activated dihedral rotations. As a result, the internal friction time scale is predominantly controlled by the height of the dihedral barriers associated with such hindered rotations. Moreover, our analysis suggests that the relatively large internal friction observed under physiological conditions may arise from concerted rotations involving more than one dihedral angle and that its magnitude is controlled by the typical length of the cooperative segment that undergoes a correlated move. These conclusions are further consistent with the observation that no (or very little) internal friction is present in the dynamics of rather flexible Gly-Ser repeat peptides ${ }^{12,38}$ and further suggests the possibility that internal friction can be controlled experimentally through varying the dihedral preferences of the polypeptide chain, which awaits its experimental validation. In view of the rather generic character of the correlated rotations mechanism, the molecular insights developed here may further be applicable to other polymeric systems.

In agreement with refs 6,7 , and 34 , the internal friction contribution to the protein's reconfiguration time is small at high denaturant and increases with decreasing denaturant concentration, becoming a dominant mechanism in the collapsed, molten-globule state characteristic of physiological conditions. As correlated rotations are expected to govern a protein's conformational search in this regime, they likely make an important contribution to the diffusion constant in the Kramers rate of protein folding. ${ }^{10,39}$ However, as the protein chain commits to folding and starts crossing the free energy barrier, additional sources of roughness may arise. ${ }^{14}$ Indeed, recent experimental evidence suggests that internal friction may be highly localized in the vicinity of a transition state. ${ }^{7}$ The effect of internal friction on folding is likely to be strongly dependent on the protein in question as, e.g., other studies showed no significant internal friction effect on the folding rate. $^{40}$ Likewise, internal friction in folded proteins, as manifested in enzymatic reactions and allosteric transitions, is likely to depend on the specific protein and specific reaction pathway, and conclusions from one protein may not be easily transferred to other proteins. ${ }^{41,42}$ In contrast, internal friction mechanisms in the unfolded state are likely to be more universal and transferrable to other proteins including those intrinsically disordered in the native state.

Our study further confirms that simple polymer models, such as CRIF, ${ }^{6,12}$ can be used to account for many essential features of the dynamics of the unfolded state and, when combined with 
MD simulations, to gain important mechanistic insights. Such models, however, cannot account for sequence-specific phenomena. Moreover, correlated dihedral rotations, such as the ones observed in this work, may be coupled to the solvent viscosity in complicated ways depending on the chain compactness. As this effect is not captured by RIF-like models, it should be further investigated by, e.g., taking into account the "protein-specific" character of these polymers, in particular, by incorporating native and non-native interactions via the energy landscapes corresponding to globular and intrinsically disordered proteins. $^{43-46}$ In particular, such interactions are expected to play an important role in protein folding during the barrier crossing dynamics, which should be explored in further studies.

\section{ASSOCIATED CONTENT}

\section{S Supporting Information}

Further details of the data analysis and the derivation of the compacted Rouse model with internal friction (CRIF). This material is available free of charge via the Internet at http:// pubs.acs.org.

\section{AUTHOR INFORMATION}

\section{Corresponding Author}

gpapoian@umd.edu

Notes

The authors declare no competing financial interest.

\section{ACKNOWLEDGMENTS}

We are grateful to Benjamin Schuler for insightful discussions. Financial support from the National Science Foundation (NSF) (grants CHE-0848571 and 1266380 to D.E.M and CAREER Award CHE-0846701 to G.A.P), the Robert A. Welch Foundation (grant F-1514) to D.E.M and the University of Maryland to G.A.P and I.E are gratefully acknowledged. CPU resources were provided by the Texas Advanced Computing Center (TACC).

\section{REFERENCES}

(1) Yang, W. Y.; Gruebele, M. Nature 2003, 423, 193-197.

(2) Kubelka, J.; Hofrichter, J.; Eaton, W. A. Curr. Opin. Struct. Biol. 2004, 14, 76-88.

(3) Bieri, O.; Wirz, J.; Hellrung, B.; Schutkowski, M.; Drewello, M.; Kiefhaber, T. Proc. Natl. Acad. Sci. U.S.A. 1999, 96, 9597-9601.

(4) Pabit, S. A.; Roder, H.; Hagen, S. J. Biochemistry 2004, 43, $12532-12538$

(5) Cellmer, T.; Henry, E. R.; Hofrichter, J.; Eaton, W. A. Proc. Natl. Acad. Sci. U.S.A. 2008, 105, 18320-18325.

(6) Soranno, A.; Buchli, B.; Nettels, D.; Cheng, R. R.; Muller-Spath, S.; Pfeil, S. H.; Hoffmann, A.; Lipman, E. A.; Makarov, D. E.; Schuler, B. Proc. Natl. Acad. Sci. U.S.A. 2012, 109, 17800-17806.

(7) Borgia, A.; Wensley, B. G.; Soranno, A.; Nettels, D.; Borgia, M. B.; Hoffmann, A.; Pfeil, S. H.; Lipman, E. A.; Clarke, J.; Schuler, B. Nat. Commun. 2012, 3, 1195.

(8) Schulz, J. C. F.; Schmidt, L.; Best, R. B.; Dzubiella, J.; Netz, R. R. J. Am. Chem. Soc. 2012, 134, 6273-6279.

(9) Zwanzig, R. Proc. Natl. Acad. Sci. U.S.A. 1988, 85, 2029-2030.

(10) Best, R. B.; Hummer, G. Phys. Rev. Lett. 2006, 96, 228104.

(11) Best, R. B.; Hummer, G. Proc. Natl. Acad. Sci. U.S.A. 2010, 107, $1088-1093$.

(12) Cheng, R. R.; Hawk, A. T.; Makarov, D. E. J. Chem. Phys. 2013, 138, 074112.

(13) Bazúa, E. R. Williams, M. C. J. Chem. Phys. 1973, 59, 28582868.
(14) Portman, J. J.; Takada, S.; Wolynes, P. G. J. Chem. Phys. 2001, 114, 5082-5096.

(15) Khatri, B. S.; Kawakami, M.; Byrne, K.; Smith, D. A.; McLeish, T. C. B. Biophys. J. 2007, 92, 1825-1835.

(16) Khatri, B. S.; Mcleish, T. C. B. Macromolecules 2007, 40, 67706777.

(17) Kuhn, W.; Kuhn, H. Helv. Chim. Acta 1945, 28, 1533-1579.

(18) De Gennes, P.-G. Scaling Concepts in Polymer Physics; Cornell University Press: Ithaca, NY, 1979.

(19) Wittebort, R. J.; Szabo, A. J. Chem. Phys. 1978, 69, 1722.

(20) Hess, B.; Kutzner, C.; van der Spoel, D.; Lindahl, E. J. Chem. Theory Comput. 2008, 4, 435-447.

(21) Daun, Y.; Wu, C.; Chowdhury, S.; Lee, M. C.; Xiong, G.; Zhang, W.; Yang, R.; Cieplak, P.; Luo, R.; Lee, T.; Caldwell, J.; Wang, J.; Kollman, P. J. Comput. Chem. 2003, 24, 1999-2012.

(22) Kremer, W.; Schuler, B.; Harrieder, S.; Geyer, M.; Gronwald, W.; Welker, C.; Jaenicke, R.; Kalbitzer, H. R. Eur. J. Biochem. 2001, 268, 2527-2539.

(23) Darden, T.; York, D.; Pedersen, L. J. Chem. Phys. 1993, 98, 10089-10092.

(24) Hess, B. J. Chem. Theory Comput. 2008, 4, 116-122.

(25) Lin, I.-C.; Tuckerman, M. E. J. Phys. Chem. B 2010, 114, 1593540.

(26) Lindorff-Larsen, K.; Trbovic, N.; Maragakis, P.; Piana, S.; Shaw, D. E. J. Am. Chem. Soc. 2012, 134, 3787-3791.

(27) Merchant, K. A.; Best, R. B.; Louis, J. M.; Gopich, I. V.; Eaton, W. A. Proc. Natl. Acad. Sci. U.S.A. 2007, 104, 1528-1533.

(28) Fitzgerald, J. E.; Jha, A. K.; Sosnick, T. R.; Freed, K. F. Biochemistry 2007, 46, 669-682.

(29) Li, D.-W.; Meng, D.; Bruschweiler, R. J. Am. Chem. Soc. 2009, 131, 14610-14611.

(30) Buscaglia, M.; Lapidus, L. J.; Eaton, W. A.; Hofrichter, J. Biophys. J. 2006, 91, 276-88.

(31) Helfand, E. J. Chem. Phys. 1971, 54, 4651-4661.

(32) García, A. E. Phys. Rev. Lett. 1992, 68, 2696-2700.

(33) Makarov, D. E. J. Chem. Phys. 2013, 138, 014102.

(34) Alexander-Katz, A.; Wada, H.; Netz, R. R. Phys. Rev. Lett. 2009, 103, 028102.

(35) Nettels, D.; Hoffmann, A.; Schuler, B. J. Phys. Chem. B 2008, 112, 6137-6146.

(36) Schuler, B.; Eaton, W. A. Curr. Opin. Struct. Biol. 2008, 18, 1626.

(37) Kurnik, M.; Hedberg, L.; Danielsson, J.; Oliveberg, M. Proc. Natl. Acad. Sci. U.S.A. 2012, 109, 5705-10.

(38) Moglich, A.; Joder, K.; Kiefhaber, T. Proc. Natl. Acad. Sci. U.S.A. 2006, 103, 12394-12399.

(39) Kramers, H. A. Physica 1940, 7, 284-304.

(40) Plaxco, K. W.; Baker, D. Proc. Natl. Acad. Sci. U.S.A. 1998, 95, 13591-13596.

(41) Zhuravlev, P. I.; Papoian, G. A. Curr. Opin. Struct. Biol. 2010, 20, 16-22.

(42) Rauscher, A.; Derényi, I.; Gráf, L.; Málnási-Csizmadia, A. IUBMB Life 2013, 65, 35-42.

(43) Sagnella, D. E.; Straub, J. E.; Thirumalai, D. J. Chem. Phys. 2000, 113, 7702-7711.

(44) Onuchic, J. N.; Wolynes, P. G. Curr. Opin. Struct. Biol. 2004, 14, $70-75$.

(45) Papoian, G. A. Proc. Natl. Acad. Sci. U.S.A. 2008, 105, 1423714238.

(46) Potoyan, D. A.; Papoian, G. A. J. Am. Chem. Soc. 2011, 133, $7405-7415$. 